\title{
Efficient Wideband DOA Estimation through Function Evaluation Techniques
}

\author{
J. Selva
}

\begin{abstract}
This paper presents an efficient evaluation method for the functions involved in the computation of direction-ofarrival (DOA) estimators. The method is a combination of the Chebyshev and barycentric interpolators, and makes use of the Discrete Cosine Transform (DCT). We present two applications of this method. The first is for reducing the complexity of the line searches in three wideband DOA estimators: Incoherent Multiple Signal Classification (IC-MUSIC), Test of Orthogonality of Projected Subspaces (TOPS), and Deterministic Maximum Likelihood (DML). And the second application is a procedure to compress the wideband DML cost function, so that it is formed by just a few summands. This compression entails a reduction in complexity by a large factor. The evaluation method and its applications are numerically assessed in several numerical examples.
\end{abstract}

\section{INTRODUCTION}

The estimation of the directions of arrival (DOAs) to an array of sensors is a fundamental problem in signal processing, which has been studied in multitude papers during the last decades. Most of the existing estimators are based on the socalled narrowband assumption, i.e, on considering that the maximum delay along the array is much smaller than the inverse bandwidths of the incoming signals, [1, Sec. 2.2]. As is well known, this assumption greatly simplifies the modeling, because the geometry of a given impinging wave just translates into a single complex factor for each sensor-wave pair. Actually, the various estimation methods in array processing can be viewed as effective ways to exploit this single-factor structure, [1]. In practice, however, the signals' bandwidths are often too wide and, as a consequence, the array geometry affects separate spectral components differently. Thus, in this wideband case there is a factor for each combination of sensor, wave and spectral component, and this fact complicates the estimation significantly.

In the literature, the basic approach for the wideband DOA problem is based on decomposing the impinging signals into a sum of components, for which the narrowband DOA models are sufficiently accurate. Then, the main problem is how the components' models should be combined in order to derive a single wideband DOA estimator. For this combination, there are two main trends in the literature. The combination can be either coherent $[2]-[5]$, in the sense that it incorporates the array snapshots from separate components into a single covariance matrix, or incoherent, meaning that a narrowband estimate is computed for each component first, and then these last estimates are combined through a method like a weighted average, [6]. Besides, there exist methods that share features of the coherent and incoherent approaches like the Test of Orthogonality of Projected Subspaces (TOPS) in [7], [8]. All these methods have been surveyed in several references, [7], [9, Ch. 3] and [10, Sec. 4.3].

We must additionally mention that the direct estimation through well-established methodologies like the maximum likelihood (ML) principle has also been attempted in a number of references. The deterministic and stochastic ML estimators have been analyzed in [11] theoretically. An approximate ML estimator has been proposed in [12], [13] and the ML estimator for near-field sources has been analyzed in [14]. The main drawback of these direct approaches is their complexity, given that the cost function has one summand for each component, and the number of components is usually high. Finally, group sparsity methods have been applied to wideband DOA estimation in [15], and low complexity implementations for these methods have been presented in [16]. See also [17].

A feature of most narrowband and wideband DOA estimators is the repeated evaluation of complicated functions, and such evaluations produce a large portion of the total computational burden. In the narrowband case, a typical example of this is the location of peaks in the Multiple Signal Classification (MUSIC) estimator [18], in which the pseudo-spectrum must be evaluated in a fine grid. A similar task is the addition of one DOA in the Generalized Likelihood Ratio Test (GLRT) detection scheme for the Deterministic ML (DML) estimator, which is based on locating the global maximum of a residual cost function [19]. We also find this feature in wideband DOA estimators like Incoherent MUSIC (IC-MUSIC) and TOPS. Additionally, the wideband case involves a repeated function evaluation at a more abstract level, that multiplies the computational burden by a large factor. Specifically, in wideband DOA estimation, the array response or some related function varies with the temporal frequency variable, and such function must be evaluated at a large number of frequencies $R$. $R$ must be large in order to control the mismatch produced by the Fast Fourier Transform (FFT). We can see this in ICMUSIC and TOPS, where the array response matrix is sampled in a grid covering the signals' spectra, in order to decompose the wideband problem into a collection of narrowband ones. Also, there is a similar evaluation in the wideband DML cost function, given that it consists of the sum of $R$ narrowband equivalents.

Thus, a common complexity issue in DOA estimation is the evaluation of various complicated functions at a large number of abscissas. Besides, these functions usually change "on the fly", because they depend on acquired data that vary for each new estimate computation. In this paper, we propose to address this complexity issue through interpolation techniques and, fundamentally, through the Chebyshev interpolation scheme. Though other interpolation schemes are usable, we restrict 
ourselves to this one for three reasons. The first is that the Chebyshev interpolator has a performance close to that of the minimax polynomial [20, Sec. 6.5]. This implies that a reasonably small number of function evaluations ensures a low interpolation error. The second is that it requires no special structure in the interpolated function and, therefore, is applicable to any sensor array or function structure. And the third is that it can be efficiently evaluated through efficient techniques like the FFT-based Discrete Cosine Transform (DCT) and the barycentric interpolator [21].

We present in this paper two contributions that provide significant complexity savings, based on interpolation techniques. The first applies to the usual line search or evaluation tasks in DOA estimation, that must be performed on highcomplexity functions. Fundamentally, it consists of an efficient method for performing such tasks (evaluation, scanning, peak location) from a reasonably small number of function evaluations. The second contribution applies to the wideband DML estimator. This estimator is optimal statistically [reaches the Cramer Rao (CR) bound at high signal-to-noise (SNR) ratios] and, therefore, its expected root-mean square (RMS) error is significantly smaller than that of sub-optimal estimators like IC-MUSIC or TOPS. Besides, it works for either uncorrelated or correlated sources and is immune to uneven spectral power distributions. However, the DML estimator is not usable in practice due to the large number of components in its cost function. We present a technique to reduce such large number to a small one, which is independent of the actual FFT length employed. The method is based on approximating the projection matrix in the DML cost function using its Chebyshev interpolator. With this interpolation method, the complexities of computing the narrowband and the wideband DML estimates just differ in a small factor (from 4 to 7 in the numerical examples).

The paper has been divided into three main parts. The first is composed by Secs. III and III, where we respectively present the signal model for the wideband DOA estimation problem and three estimators: IC-MUSIC, TOPS, and Direct DML. The main purpose of this part is to present state-ofart estimators involving function evaluations or line searches as those just commented. The second part is Sec. IV], where we address the problem of efficiently evaluating the typical functions appearing in DOA estimation with low complexity. By low complexity, we mean two features: the number of function evaluations is small and, at the same time, it is possible to obtain the function's value at arbitrary abscissas in a small number of arithmetic operations. We achieve this low complexity through a combination of techniques, that include the Chebyshev and barycentric interpolators, and the DCT. The third part consists of four sections, in which we exploit the interpolation method in the second part in order to reduce the complexity of the estimators in the first (IC-MUSIC, TOPS, and Direct DML). In Sec. V, we reduce the complexity of the line searches involved in the estimators. In Sec. VI, we show how the number of summands in the DML cost function can be drastically reduced through interpolation. Finally, we discuss the reductions in complexity that can be expected in Sec. VII and evaluate the methods in this third part in Sec.
VIII numerically.

\section{A. Notation}

The notation is the following:

- We write vectors and matrices in lower and upper bold face respectively. So $\boldsymbol{y}$ denotes a vector and $\boldsymbol{Y}$ a matrix.

- $I$ denotes the identity matrix.

- $[\boldsymbol{y}]_{k}$ and $[\boldsymbol{Y}]_{p, q}$ represent the $k$ th and $(p, q)$ elements of $\boldsymbol{y}$ and $\boldsymbol{Y}$ respectively.

- The vector formed by appending a scalar $a$ to a column vector $\boldsymbol{y}$ is denoted $[\boldsymbol{y} ; a]$.

- ' $\odot$ ' is the element-by-element product of two equal-size matrices or vectors.

- $\boldsymbol{Y}^{\dagger}$ denotes the pseudo-inverse of matrix $\boldsymbol{Y}$. As is well known, $\boldsymbol{Y}^{\dagger}=\left(\boldsymbol{Y}^{H} \boldsymbol{Y}\right)^{-1} \boldsymbol{Y}^{H}$ if $\boldsymbol{Y}$ has full-column rank.

- New symbols and functions are introduced using the operator ' $\equiv$ '.

- $T_{p}(x)$ denotes the Chebyshev polynomial of order $p$, defined by

$$
T_{p}(x) \equiv \cos (p \arccos (x)), p=0,1,2, \ldots
$$

\section{SignAl MODEL FOR WIDEBAND DOA ESTIMATION}

Consider a linear array of $M$ sensors at positions $d_{m}$, into which $K$ wideband signals impinge with angles of arrival $\theta_{k}$ relative to the broadside, $(m=1, \ldots, M, k=1, \ldots, K)$. We assume that these signals are passband with spectra contained in $\left[f_{o}-B / 2, f_{o}+B / 2\right]$, where $B>0$ and $f_{o}>B / 2$. After demodulation from frequency $f_{o}$ to baseband, the signal from the $m$ th sensor follows the model

$$
x_{m}(t)=\sum_{k=1}^{K} e^{-j 2 \pi f_{o} \tau_{m} \gamma_{k}} b_{m}\left(\gamma_{k}\right) s_{k}\left(t-\tau_{m} \gamma_{k}\right)+w_{m}(t),
$$

where

- $\tau_{m}$ is the delay between the array's reference point and the $m$ th sensor at the propagation velocity,

$$
\left.\tau_{m} \equiv \frac{d_{m}}{c}, \text { (propagation velocity } c\right)
$$

- $\gamma_{k}$ is the sine of the $k$ th angle of arrival,

$$
\gamma_{k} \equiv \sin \theta_{k}
$$

- $b_{m}(\gamma)$ is the $m$ th sensor pattern,

- $s_{k}(t)$ is the lowpass equivalent of the $k$ th impinging signal,

- and the $w_{m}(t)$ are independent complex white noise processes of equal variance.

Next, we assume the receiver takes regular samples of each $x_{m}(t)$ with a period $T$ fulfilling the Nyquist condition $(B T<$ 1 ) in $J$ slots of $N$ samples each. The receiver then computes the $N$-length DFT of each slot sequence, producing spectral samples denoted $\tilde{x}_{m, \ell}(r /(N T))$, where $r$ and $\ell$ are the frequency and slot indices respectively. The ranges for these indices are $\ell=1,2, \ldots, J$ and $r=r_{1}, r_{1}+1, \ldots, r_{1}+R-1$, where $r_{1}$ is the first frequency with significant signal power 
in a sequence of $R$ frequencies. If $N$ is sufficiently large, $\tilde{x}_{m, \ell}(r /(N T))$ follows the approximate model

$$
\begin{array}{r}
\tilde{x}_{m, \ell}\left(\frac{r}{N T}\right)=\sum_{k=1}^{K} e^{-j 2 \pi\left(f_{o}+r /(N T)\right) \tau_{m} \gamma_{k}} b_{m}\left(\gamma_{k}\right) \tilde{s}_{k, \ell}\left(\frac{r}{N T}\right) \\
+\tilde{w}_{m, \ell}\left(\frac{r}{N T}\right),
\end{array}
$$

where $\tilde{s}_{k, \ell}(r /(N T))$ and $\tilde{w}_{m}(r /(N T))$ have the obvious definitions. In vector notation, this model reads

$$
\boldsymbol{X}_{r}=\boldsymbol{A}(r, \gamma) \boldsymbol{S}_{r}+\boldsymbol{W}_{r},
$$

where

$$
\begin{aligned}
& {\left[\boldsymbol{X}_{r}\right]_{m, \ell} \equiv \tilde{x}_{m, \ell}\left(\frac{r}{N T}\right),[\gamma]_{k} \equiv \gamma_{k}} \\
& {[\boldsymbol{a}(r, \gamma)]_{m} \equiv e^{-j 2 \pi\left(f_{o}+r /(N T)\right) \tau_{m} \gamma} b_{m}(\gamma),} \\
& {[\boldsymbol{A}(r, \gamma)]_{, k} \equiv \boldsymbol{a}\left(r, \gamma_{k}\right),\left[\boldsymbol{S}_{r}\right]_{k, \ell} \equiv \tilde{s}_{k, \ell}\left(\frac{r}{N T}\right),} \\
& {\left[\boldsymbol{w}_{r}\right]_{m, \ell} \equiv \tilde{w}_{m}\left(\frac{r}{N T}\right),} \\
& m=1, \ldots, M, \quad k=1, \ldots, K, \quad \ell=1, \ldots, J
\end{aligned}
$$

In the next section, we present the IC-MUSIC, TOPS, and Direct DML estimators for this model.

\section{Selection of Wideband DOA estimators}

Given the sample covariance matrices

$$
\boldsymbol{R}_{r} \equiv \boldsymbol{X}_{r} \boldsymbol{X}_{r}^{H},
$$

the IC-MUSIC estimates are the angles corresponding to the $K$ main local minima of the cost function

$$
L_{M U}(\gamma) \equiv \sum_{r=r_{1}}^{r_{1}+R-1} \boldsymbol{a}(r, \gamma){ }^{H} \boldsymbol{V}_{r} \boldsymbol{V}_{r}^{H} \boldsymbol{a}(r, \gamma),
$$

where $\boldsymbol{V}_{r}$ spans the noise subspace of $\boldsymbol{R}_{r} ;\left(\boldsymbol{V}_{r}\right.$ is composed of the $M-K$ eigenvectors with smaller eigenvalues). (4) is a natural extension of the corresponding narrowband estimator, which consists of a single summand [18].

Another subspace method is TOPS [7]. In this estimator, the noise subspaces from $R-1$ frequencies are combined into a single matrix that depends on a DOA parameter $\gamma$. Then, the parameter values for which the matrix approximately becomes rank deficient correspond to the angle-of-arrival estimates. In order to introduce this estimator, define first the $R-1$ matrices

$$
\boldsymbol{E}_{r}(\gamma) \equiv \boldsymbol{U}_{r^{\prime}}^{H} \operatorname{diag}\left(\boldsymbol{a}\left(r-r_{1}, \gamma\right)^{*}\right) \boldsymbol{V}_{r},
$$

for $r=r_{1}, r_{1}+1, \ldots, r_{1}+R-1, r \neq r^{\prime}$, where $\boldsymbol{U}_{r^{\prime}}$ is the matrix spanning the signal subspace at a fixed $r^{\prime}$, and $\boldsymbol{V}_{r}$ was already defined for (4). $r^{\prime}$ is chosen so that the power $\operatorname{tr}\left\{\boldsymbol{R}_{r^{\prime}}\right\}$ is sufficiently high. $\left(\boldsymbol{U}_{r^{\prime}}\right.$ contains the eigenvectors associated with the $K$ larger eigenvalues of $\boldsymbol{R}_{r^{\prime}}$.) Next, stack these $R-1$ matrices into a single one,

$$
\boldsymbol{E}(\gamma) \equiv\left[\boldsymbol{E}_{r_{1}}(\gamma), \boldsymbol{E}_{r_{1}+1}(\gamma), \ldots, \boldsymbol{E}_{r_{1}+R-1}(\gamma)\right],
$$

where the block $\boldsymbol{E}_{r^{\prime}}(\gamma)$ is missing. The TOPS estimates are the $K$ main local minima of the cost function

$$
L_{T}(\gamma) \equiv \sigma_{\min }(\boldsymbol{E}(\gamma)),
$$

where $\sigma_{\min }(\cdot)$ denotes the smallest singular value of the given matrix.

Next, let us introduce the DML estimator, termed "Direct DML" in the rest of the paper. For the model in (2), the Direct DML estimate of $S$ and $\gamma$ is given by the arguments minimizing the cost function

$$
L_{M L, 0}\left(\boldsymbol{S}_{r_{1}}, \ldots, \boldsymbol{S}_{r_{1}+R-1}, \gamma\right) \equiv \sum_{r=r_{1}}^{r_{1}+R-1}\left\|\boldsymbol{X}_{r}-\boldsymbol{A}(r, \boldsymbol{\gamma}) \boldsymbol{S}_{r}\right\|_{F}^{2}
$$

As is well known, each summand in this cost function can be independently minimized in $\boldsymbol{S}_{r}$ for fixed $\gamma$, using the pseudo-inverses of the matrices $\boldsymbol{A}(r, \boldsymbol{\gamma})$. If the resulting $\boldsymbol{S}_{r}$ are substituted into (6), then we obtain the compressed cost function,

$$
L_{M L}(\gamma) \equiv \sum_{r=r_{1}}^{r_{1}+R-1} \operatorname{tr}\left\{\boldsymbol{P}_{\perp}(r, \gamma) \boldsymbol{R}_{r}\right\}
$$

where $\boldsymbol{P}_{\perp}(r, \gamma)$ denotes the projection matrix for the orthogonal complement of $\boldsymbol{A}(r, \gamma)$,

$$
\boldsymbol{P}_{\perp}(r, \gamma) \equiv \boldsymbol{I}-\boldsymbol{A}(r, \gamma) \boldsymbol{A}(r, \gamma)^{\dagger} .
$$

The Direct DML estimator of $\gamma$ is the vector minimizing $L_{M L}(\gamma)$.

In the sequel, we present a detection-estimation method for computing this estimate, that resembles well-known procedures for the narrowband case, like those in [22], [23] and [24. Sec. 4.6)]. It is a combination of two steps, one for detecting additional components, and another for refining a given estimate. The first is a variant of the Generalized Likelihood Ratio (GLR) test in [25], and the second is the modified variable projection method (MVP) in [26], which is an iterative method that converges in a small number of iterations.

The method operates on a given estimate $\gamma_{K, \alpha}$, repeating in turn a detection step followed by an estimation step, until a statistical test fails. The sub-indices $K$ and $\alpha$ in $\gamma_{K, \alpha}$ are the length of this same vector and the number of iterations in the estimation step respectively. The initial vector $\gamma_{0,0}$ is empty. Both steps are described in the next two sub-sections.

\section{A. Detection step for the wideband DML estimator}

Given an iterate $\gamma_{K, \alpha}$ (which can be initially empty), the detection method decides whether to look for an additional parameter $\gamma$ and, if so, selects as $\gamma$ the minimum of $L_{M L}\left(\left[\gamma_{K, \alpha} ; \gamma\right]\right)$. The detection is based on a statistical test for $L_{M L}\left(\gamma_{K, \alpha}\right)$, assuming that $\gamma_{K, \alpha}$ is the true parameter vector. More precisely, if $\gamma_{K, \alpha}$ is the true vector and there is available a noise variance estimate $\hat{\sigma}^{2}$, then $L_{M L}\left(\gamma_{K, \alpha}\right) / \hat{\sigma}^{2}$ approximately follows a $\chi^{2}$ distribution with $2(M-K) R$ degrees of freedom. Thus, an additional component is sought if $L_{M L}\left(\gamma_{K, \alpha}\right)>A$, with

$$
A \equiv \frac{\hat{\sigma}^{2}}{2} F^{-1}\left(1-P_{F A}\right),
$$

where $F$ is the $\chi^{2}$ cumulative distribution function, and $P_{F A}$ a fixed false-alarm probability. 
We may summarize this detection step as follows. If $L_{M L}\left(\gamma_{K, \alpha}\right)<A$, the iterative process finishes and the final estimate is $\gamma_{K, \alpha}$, but if $L_{M L}\left(\gamma_{K, \alpha}\right)>A$ then it proceeds to the estimation step with the new vector $\gamma_{K+1,0}=\left[\gamma_{K, \alpha} ; \gamma\right]$, where

$$
\gamma=\arg \min _{\gamma^{\prime}} L_{M L}\left(\left[\gamma_{K, \alpha} ; \gamma^{\prime}\right]\right) .
$$

\section{B. Estimation step for the wideband DML estimator}

This step is an implementation of the MVP method in [26], already used in [23], [27] and [24, Sec. 4.6)] for the narrowband problem. For introducing it, let us define first the following matrix of differentials with the same size as $\boldsymbol{A}(r, \gamma)$,

$$
[\boldsymbol{D}(r, \boldsymbol{\gamma})]_{m, k} \equiv \frac{d}{d \gamma_{k}}[\boldsymbol{A}(r, \boldsymbol{\gamma})]_{m, k} .
$$

Its explicit expression can be readily computed from (3). Also, it is convenient to define the following shorthand notation

$$
\begin{aligned}
\boldsymbol{A}_{r, K, \alpha} & \equiv \boldsymbol{A}\left(r, \gamma_{K, \alpha}\right), \boldsymbol{D}_{r, K, \alpha} \equiv \boldsymbol{D}\left(r, \gamma_{K, \alpha}\right), \\
\boldsymbol{P}_{\perp, r, K, \alpha} & \equiv \boldsymbol{P}_{\perp}\left(r, \gamma_{K, \alpha}\right) .
\end{aligned}
$$

Given an iterate $\gamma_{K, \alpha}$, the MVP method refines it using the iteration

$$
\gamma_{K, \alpha+1}=\gamma_{K, \alpha}-\mu \boldsymbol{H}_{K, \alpha}^{-1} \boldsymbol{g}_{K, \alpha},
$$

where usually $\mu=1$, though $0<\mu<1$ may be used to ensure a cost function decrease [28. Ch. 5], and $\boldsymbol{g}_{K, \alpha}$ and $\boldsymbol{H}_{K, \alpha}$ are the gradient and approximate Hessian of (7) at $\gamma=\gamma_{K, \alpha}$,

$$
\begin{gathered}
\boldsymbol{g}_{K, \alpha} \equiv-2 \operatorname{Re}\left\{\operatorname{diag}\left\{\sum_{r=r_{1}}^{r_{1}+R-1} \boldsymbol{A}_{r, K, \alpha}^{\dagger} \boldsymbol{R}_{r} \boldsymbol{P}_{\perp, r, K, \alpha} \boldsymbol{D}_{r, K, \alpha}\right\}\right\} \\
\boldsymbol{H}_{K, \alpha} \equiv 2 \operatorname{Re}\left\{\sum_{r=r_{1}}^{r_{1}+R-1}\left(\boldsymbol{D}_{r, K, \alpha}^{H} \boldsymbol{P}_{\perp, r, K, \alpha} \boldsymbol{D}_{r, K, \alpha}\right\}\right)^{T} \\
\left.\odot\left(\boldsymbol{A}_{r, K, \alpha}^{\dagger} \boldsymbol{R}_{r}\left(\boldsymbol{A}_{r, K, \alpha}^{\dagger}\right)^{H}\right)\right\} .
\end{gathered}
$$

The convergence rate of 9 is quadratical close to a minimum, and usually a small number of iterations is sufficient. This gradient and approximate Hessian can be efficiently computed from the Housholder QR decompositions of the matrices $\boldsymbol{A}_{p, K, \alpha}$, as shown in [24, Sec. 4.6.4b)]. Actually, it is not necessary to compute neither $\boldsymbol{A}_{p, K, \alpha}^{\dagger}$ nor $\boldsymbol{P}_{\perp, p, K, \alpha}$. The iteration in (9) is repeated until there is no significant reduction in the cost function's value. Afterward, the execution proceeds to the detection step again.

\section{EFFiciENT FUNCTION EVALUATION}

A feature of the DOA estimators in the previous section, as well as of many other estimators in the literature, is that they involve the repeated evaluation of one or more functions, and such evaluations take up a large portion of the total computational burden. Let us draw some examples of such functions from the estimators presented in the previous section. For this, let $g(\lambda)$ and $[a, b]$ respectively denote, in each case, the evaluated function and its domain. We have the following examples:

- In IC-MUSIC, the $K$ main local minima of the pseudospectrum $L_{M U}(\gamma)$ in (4) must be obtained through some numerical method, and such method may involve the evaluation of $L_{M U}(\gamma)$ at many abscissas $\gamma$. So, in this case, the function's variable is $\lambda=\gamma$, its domain is $[a, b]=[-1,1]$, and the evaluated function is $g(\lambda)=L_{M U}(\lambda)$.

- In TOPs, we find a similar peak search but for the function $g(\lambda)=L_{T}(\lambda)$ in (5), where, again, we have $\lambda=\gamma$ and $[a, b]=[-1,1]$.

- In the DML estimator-detector, the addition of a new $\gamma$ involves a line search on the function $g(\lambda)=$ $L_{M L}\left(\left[\gamma_{K, \alpha} ; \lambda\right]\right)$ in 88 . And again, we have $\lambda=\gamma$ and $[a, b]=[-1,1]$.

- The Direct DML cost function in (7) contains another example of repeated function evaluation. Actually, there is one such repeated evaluation for every component of the projection matrix $\boldsymbol{P}_{\perp}(r, \gamma)$. More precisely, for arbitrary indices $m$ and $m^{\prime},\left(1 \leq m \leq M, 1 \leq m^{\prime} \leq M\right)$, the $\left(m, m^{\prime}\right)$ component of the projection matrix $\boldsymbol{P}_{\perp}(r, \gamma)$ is a function of $r$, which is repeatedly evaluated ( $R$ times) in order to compute $L_{M L}(\gamma)$, given a fixed vector $\gamma$. Thus, in this case, the function is

$$
g(\lambda)=\left[\boldsymbol{P}_{\perp}(\lambda, \gamma)\right]_{m, m^{\prime}} .
$$

with variable $\lambda=r$ and domain $[a, b]=\left[r_{1}, r_{1}+R-1\right]$.

In this paper, we propose a simple strategy for reducing the complexity of these evaluations, applicable to most practical functions $g(\lambda)$. The strategy consists of evaluating the Chebyshev interpolator of $g(\lambda)$, denoted $\tilde{g}(\lambda)$ in the sequel, rather than $g(\lambda)$ proper. For $P$ interpolation nodes, the interpolator $\tilde{g}(\lambda)$ can be written as

$$
\tilde{g}(\lambda) \equiv \sum_{n=1}^{P} g\left(\lambda_{n}\right) \phi_{n}(\lambda),
$$

where $\lambda_{n}$ and $\phi_{n}(\lambda)$ are the set of nodes and weight functions in the Chebyshev interpolation scheme respectively. $\left[\lambda_{n}\right.$ and $\phi_{n}(\lambda)$ will be defined later in (17) and (18).]

Obviously, this strategy is useful only if the following conditions hold:

1) The number of nodes $P$ in 10 must be reasonably small.

2) $\tilde{g}(\lambda)$ must be a good approximation to $g(\lambda)$.

3) There must be efficient techniques for obtaining the value of $\tilde{g}(\lambda)$ at arbitrary $\lambda$.

The first two conditions hold in most cases in practice, given that the typical functions $g(\lambda)$ belong to a specific class of smooth functions, (like the class of differentiable or analytic functions, for example), and Chebyshev interpolation performs very well for such classes [20, Sec. 6.2]. And we present in the next sub-subsections efficient methods for fulfilling condition 3 ), thus making the whole strategy viable in practice.

In the rest of this section, we present several methods related with the evaluation of $\tilde{g}(\lambda)$. First, we show in the next subsection that $\tilde{g}(\lambda)$ can be transformed into a cosine polynomial, denoted $\tilde{g}_{2}(t)$, through a change of variable. Additionally, we show that $\tilde{g}_{2}(t)$ can be efficiently evaluated in a grid of abscissas using the DCT. Afterward, we show in sub-section IV-B that if $\tilde{g}_{2}(t)$ is known in a proper grid of abscissas, then it can be evaluated at any $t$ in a small number of arithmetic 
operations by means of a barycentric interpolator. Finally, we combine all these results in Sub-sec. IV-C, where we present an efficient method for computing $\tilde{g}(\lambda)$ in a small number of arithmetic operations, thus fulfilling condition 3) above.

\section{A. Chebyshev interpolation and its relation with the DCT}

We proceed to introduce three different forms of the same Chebyshev interpolator [20, Ch. 6]. For this, let us employ two new variables, $x$ and $t$, which depend on $\lambda$ through the following invertible variable changes. $x$ is selected to vary in range $[-1,1]$ and is related with $\lambda$ through the equation

$$
\lambda=\eta(x),
$$

where $\eta$ is the linear mapping of $[-1,1]$ onto $[a, b]$ given by

$$
\eta(x) \equiv \frac{a+b}{2}+\frac{a-b}{2} x .
$$

And in turn, $t$ is related with $x$ through the equation

$$
x=\cos (\pi t),
$$

and $t$ varies in the range $[0,1]$.

(11) and (13) allow us to define two new functions from $g(\lambda)$, denoted $g_{1}(x)$ and $g_{2}(t)$,

$$
\begin{aligned}
g_{1}(x) & \equiv g(\eta(x)), \\
g_{2}(t) & \equiv g_{1}(\cos (\pi t))=g(\eta(\cos (\pi t))) .
\end{aligned}
$$

Also, if $\tilde{g}_{1}(x)$ denotes the Chebyshev interpolator of $g_{1}(x)$, then the changes in 12 and 13 allow us to define interpolators for $g(\lambda)$ and $g_{2}(t)$ respectively,

$$
\tilde{g}(\lambda) \equiv \tilde{g}_{1}\left(\eta_{i}(\lambda)\right), \tilde{g}_{2}(t) \equiv \tilde{g}_{1}(\cos (\pi t)),
$$

where $\eta_{i}(\lambda)$ is the inverse of $\eta(x)$ in 12 ,

$$
\eta_{i}(\lambda) \equiv \frac{2}{a-b}\left[\lambda-\frac{a+b}{2}\right] .
$$

The Chebyshev interpolator is conventionally introduced in the $x$ domain $[-1,1]$ for $g_{1}(x)$, and is defined in two equivalent ways. In the first definition, it is viewed as the Lagrange interpolator with kernel $T_{P}(x)$,

$$
g_{1}(x) \approx \tilde{g}_{1}(x) \equiv \sum_{n=1}^{P} g_{1}\left(x_{n}\right) \frac{T_{P}(x)}{T_{P}^{\prime}\left(x_{n}\right)\left(x-x_{n}\right)},
$$

where $T_{P}^{\prime}(x)$ the derivative of $T_{P}(x)$ and $x_{n}$ is the set of roots of $T_{P}(x)$,

$$
x_{n} \equiv \cos \left(\pi \frac{2 n-1}{2 P}\right), n=1, \ldots, P .
$$

And in the second definition, the Chebyshev polynomials are viewed as elements of a vector space and it consists of two basic formulas. The first expresses the interpolator $\tilde{g}_{1}(x)$ as an element of the linear space spanned by the polynomials $T_{p}(x)$. The formula is

$$
g_{1}(x) \approx \tilde{g}_{1}(x) \equiv \sum_{p=0}^{P-1} c_{p} T_{p}(x)
$$

where the prima $\left({ }^{\prime}\right)$ means that the summand for $p=0$ must be divided by 2 , and $c_{p}$ is a set of coefficients. And the second formula states the fact that the Chebyshev polynomials are orthogonal when restricted to the abscissas $x_{n}$, and allow us to compute the coefficients $c_{p}$,

$$
c_{p} \equiv \frac{2}{P} \sum_{n=1}^{P} g_{1}\left(x_{n}\right) T_{p}\left(x_{n}\right)
$$

All these formulas, specially (14), (15) and (16), have their equivalents in the $\lambda$ and $t$ domains, which can be readily obtained using the variable changes in (11) and (13). We will require in the rest of the paper the equivalents of the interpolation nodes $x_{n}$ in the $\lambda$ and $t$ domains,

$$
\lambda_{n} \equiv \eta\left(x_{n}\right), t_{n} \equiv \frac{2 n-1}{2 P}, \quad n=1, \ldots, P
$$

and the equivalent of the Lagrange formula in 14 in the $\lambda$ domain, which is 10 with weight functions given by

$$
\phi_{n}(\lambda) \equiv \frac{T_{P}\left(\eta_{i}(\lambda)\right)}{T_{P}^{\prime}\left(\eta_{i}\left(\lambda_{n}\right)\right)\left(\eta_{i}(\lambda)-\eta_{i}\left(\lambda_{n}\right)\right)} .
$$

An interesting link between Chebyshev and trigonometric interpolation is the fact that the equivalents of $(15)$ and $[16)$ in the $t$ domain are cosine sums, that can be efficiently evaluated using the discrete cosine transform (DCT). Specifically, since

$$
T_{p}(x)=T_{p}(\cos (\pi t))=\cos (\pi p t),
$$

we have that $(15)$ translates into a cosine sum

$$
g_{2}(t) \approx \tilde{g}_{2}(t) \equiv \sum_{p=0}^{P-1} c_{p} \cos (\pi p t) .
$$

Besides, since $t_{n}$ varies through the regular sampling grid in (17), we have that (19), restricted to the sampling points $t_{n}$, is a type-3 DCT,

$$
\tilde{g}_{2}\left(t_{n}\right)=\sum_{p=0}^{P-1} c_{p} \cos \left(\pi p \frac{2 n-1}{2 P}\right)
$$

And as to the formula in 16 , since $g_{1}\left(x_{n}\right)=g_{2}\left(t_{n}\right)$, we have that its $t$-domain equivalent is a type-2 DCT,

$$
c_{p} \equiv \frac{2}{P} \sum_{n=1}^{P} g_{2}\left(t_{n}\right) \cos \left(\pi p \frac{2 n-1}{2 P}\right) .
$$

(20) and (21) allow us to oversample the sequence of values $\tilde{g}_{2}\left(t_{n}\right), n=1, \ldots, P$, by any integer factor $\alpha>1$ using a method akin to the zero-padding FFT algorithm, [29]. For this, compute $c_{p}$ using the type-2 DCT in 21, append $P(\alpha-1)$ zeros, and finally compute the type-3 DCT in 20, with $\alpha P$ in place of $P$. The outcome of these operations is the oversampled sequence $\tilde{g}_{2}\left(t_{n}^{\prime}\right)$ where

$$
t_{n}^{\prime} \equiv \frac{2 n-1}{2 \alpha P}, \quad n=1,2, \ldots, \alpha P .
$$




\section{B. Barycentric interpolation for band-limited functions}

A known fact in sampling theory is that if a band-limited function (or signal) is sampled at a rate above its Nyquist rate, then it can be interpolated from a small number of samples surrounding the evaluation abscissa with high accuracy. In more precise terms, if the signal has two-sided bandwidth $B$, is bounded by a constant $A$, and is sampled with period $T$, then there are accurate formulas that employ $2 Q+1$ samples, whose error is bounded by

$$
\frac{A}{\sinh (\pi(1-B T) Q)} \approx \frac{A}{2} e^{-\pi(1-B T) Q} .
$$

(See [30].) We can see in this formula an exponential trend, i.e, the error converges to zero exponentially with $Q$. In practice, (23) implies that we may interpolate a band-limited function with high accuracy from just a few of its values surrounding the evaluation abscissa.

In the previous section, $\tilde{g}_{2}(t)$ is a band-limited function to which such accurate formulas can be applied. Specifically, we have the following:

- From 19, $\tilde{g}_{2}(t)$ has two-sided bandwidth $P-1$.

- The oversampling method by the end of the previous section allows us to sample $\tilde{g}_{2}(t)$ above its Nyquist rate with, for example, oversampling factor $\alpha=2$ (or more) in the grid $t_{n}^{\prime}$ in 22.

- $\tilde{g}_{2}(t)$ is obviously bounded and since it approximates $g_{2}(t)$ and in turn $g(\lambda)$, any bound $A$ for $g(\lambda)$ is roughly valid for $\tilde{g}_{2}(t)$.

Among the accurate formulas, the barycentric one introduced in [21] additionally computes the interpolated value in a small number of arithmetic operations, and also gives the values of successive derivatives, if required. More precisely, it just requires 5 arithmetic operations for each input sample [roughly $5(2 Q+1)$ flops in total].

In order to introduce this interpolator for $\tilde{g}_{2}(t)$, let us extend first the definition of $t_{n}^{\prime}$ to any integer $n$, i.e, define

$$
t_{n}^{\prime} \equiv \frac{2 n-1}{2 \alpha P}, \text { integer } n .
$$

Also, let $n$ denote the index of the $t_{n}^{\prime}$ lying closest to $t$, and $u$ the shift of $t$ relative to $t_{n}^{\prime}$, but normalized to the grid spacing,

$$
u \equiv \alpha P\left(t-t_{n}^{\prime}\right)
$$

The barycentric interpolator is

$$
\tilde{g}_{2}(t) \approx \tilde{g}_{3}(t) \equiv \frac{\tilde{g}_{2}\left(t_{n}^{\prime}\right)+u \sum_{\substack{q=-Q \\ q \neq 0}}^{Q} \frac{w_{|q|}(-1)^{q} \tilde{g}_{2}\left(t_{n-q}^{\prime}\right)}{q+u}}{1+u \sum_{\substack{q=-Q \\ q \neq 0}}^{Q} \frac{w_{|q|}(-1)^{q}}{q+u}} .
$$

where $w_{q}$ is a set of samples of the Fourier transform of the Kaiser-Bessel window,

$$
w_{q} \equiv \frac{\operatorname{sinc}\left((1-1 / \alpha) \sqrt{q^{2}-(Q+1)^{2}}\right)}{\operatorname{sinc}(j(1-1 / \alpha)(Q+1))} .
$$

As can be readily seen in 24, $\tilde{g}_{3}(t)$ can be computed in a small number of operations and it interpolates $g(\lambda)$ because, if $\lambda, x$, and $t$ are related through (11) and (13), then we have

$$
g(\lambda) \approx \tilde{g}(\lambda)=\tilde{g}_{1}(x)=\tilde{g}_{2}(t) \approx \tilde{g}_{3}(t) .
$$

\section{Proposed function evaluation method}

We now combine the results in the previous two subsections in an efficient evaluation method for a generic $g(\lambda)$. The method has a set-up stage in which $g(\lambda)$ is directly evaluated, and an evaluation step in which arbitrary values of $g(\lambda)$ can be obtained through interpolation. The set-up stage consists of two steps:

1) Compute the $P$ values $g_{2}\left(t_{n}\right)$, which actually are values of $g(\lambda)$ due to 11 and 13 ,

$$
g_{2}\left(t_{n}\right)=g\left(\eta\left(\cos \left(\pi t_{n}\right)\right) .\right.
$$

In this step, $P$ is selected so that $\tilde{g}(\lambda)$ approximates $g(\lambda)$ with enough accuracy. We may expect a rather small $P$ to be sufficient, given that the Chebyshev interpolator is near-minimax, [20, Sec. 6.5].

2) Upsample the sequence $g_{2}\left(t_{n}\right)$ by factor $\alpha \geq 2$, obtaining the values of $\tilde{g}_{2}(t)$ at the $\alpha P$ abscissas $t_{n}^{\prime}$ in 22). This can be done efficiently using the DCT method explained at the end of Sub-sec. IV-A

In the evaluation stage, there are three operations that can be performed on demand with low complexity. We denote them with the acronyms $\mathbf{O p 1}, \mathbf{O p 2}$, and $\mathbf{O p 3}$ for latter reference:

Op1 Coarse localization of maxima or minima of $g(\lambda)$. This can be done by scanning the available sequence $\tilde{g}_{2}\left(t_{n}^{\prime}\right)$, given that it is also a sequence of values of $\tilde{g}(\lambda)$,

$$
\tilde{g}_{2}\left(t_{n}^{\prime}\right)=\tilde{g}\left(\eta\left(\cos \left(\pi t_{n}^{\prime}\right)\right)\right) \approx g\left(\eta\left(\cos \left(\pi t_{n}^{\prime}\right)\right)\right) .
$$

Op2 Computation of $g(\lambda)$ at an arbitrary $t$. Simply evaluate the barycentric interpolator $\tilde{g}_{3}(t)$, where

$$
t=\frac{1}{\pi} \arccos \left(\eta_{i}(\lambda)\right)
$$

Op3 Refine a coarse estimate of a local maximum or minimum of $g(\lambda)$. This can be done using Newton's method but applied to $\tilde{g}_{3}(t)$ rather than $g(\lambda)$. More precisely, if the peak is approximately at $t=\tau_{0}$ and we denote the $r$ iteration in Newton's method as $\tau_{r}$, then we may iteratively approach the local maximum through the iteration

$$
\tau_{r+1}=\tau_{r}-\frac{\tilde{g}_{3}^{\prime}\left(\tau_{r}\right)}{\tilde{g}_{3}^{\prime \prime}\left(\tau_{r}\right)},
$$

where $\tilde{g}_{3}^{\prime}\left(\tau_{r}\right)$ and $\tilde{g}_{3}^{\prime \prime}\left(\tau_{r}\right)$ are delivered by the barycentric interpolator, as explained in [21]. Usually, just three iterations suffice. If $\tau$ is the final abscissa, then the corresponding abscissa of $g(\lambda)$ is $\lambda=\eta(\cos (\pi \tau))$. 


\section{APPliCATION TO LINE SEARCHES}

We show in the sub-sections that follow the way in which the interpolation techniques in IV reduce the complexity of the line searches appearing in the estimators discussed in Sec. III]

\section{A. IC-MUSIC}

The efficient computation of the IC-MUSIC consists of particularizing the evaluation method in Sec. IV-C for the pseudo-spectrum $L_{M U}(\gamma)$ in (4). More precisely, first we identify the generic function $g(\lambda)$ in Sec. IV] with $L_{M U}(\gamma)$ in the domain $[a, b]=[-1,1]$, i.e, we take $\lambda=\gamma$ and

$$
g(\gamma)=L_{M U}(\gamma)
$$

From $[12$, , we have that for the domain $[-1,1]$ it is $\eta(x)=$ $-x$.

And second, we carry out the steps in the set-up stage in Sec. IV-C, and exploit some of the evaluation operations in that section as follows:

1) Compute the $P$ values $L_{M U}\left(-\cos \left(\pi t_{n}\right)\right), n=1, \ldots, P$, using the initial formula in (4), where $t_{n}$ was defined (17). Recalling the changes of variables in Sec. IV, these values can be interpreted as samples of $g_{2}(t)$ through the formula

$$
g_{2}\left(t_{n}\right)=L_{M U}\left(-\cos \left(\pi t_{n}\right)\right), n=1, \ldots, P,
$$

which is the translation of (25).

2) Upsample the sequence in (26) by factor $\alpha>1$, [step 2) in set-up stage], using the DCT method in Sub-sec. IV-A

3) Coarsely locate the $K$ main local minima of $g(\lambda)$ using Op1.

4) Refine each of the previous local minima using Op3. The resulting values are the final IC-MUSIC estimates.

\section{B. TOPS}

The computation of the TOPS estimates is identical to that of the IC-MUSIC estimates just explained, but replacing $L_{M U}(\gamma)$ with $L_{T}(\gamma)$, [defined in [5] ].

\section{Minimization of $L_{M L}\left(\left[\gamma_{K, \alpha} ; \gamma\right]\right)$ in 8}

This minimization is performed in the $\gamma$ variable only and is required in the detection step for the DML estimator in SubSec. III-A. It can be computed using the method explained in Sub-sec. V-A for IC-MUSIC. The only difference is that we only require the global minimum of $L_{M L}\left(\left[\gamma_{K, \alpha} ; \gamma\right]\right)$ rather than a number of its main local minima.

\section{REDUCTION OF THE NUMBER OF DML COST FUNCTION SUMMANDS}

Let us see how the DML cost function in (7) can be compressed, so that its large number of summands $R$ is ruduced to a small number $P$. For this, notice three key features of (7). The first is that $L_{M L}(\gamma)$ depends linearly on the projection matrices $\boldsymbol{P}_{\perp}(r, \gamma)$. The second is that $\boldsymbol{P}_{\perp}(r, \gamma)$ is evaluated a large number of times $R$, given that $R$ must be large in order to obtain a small FFT mismatch. And the third is that $\boldsymbol{P}_{\perp}(r, \gamma)$ varies smoothly with $r$, given that to pass from a given frequency $r /(N T)$ to its nearby frequency $(r+1) /(N T)$ produces a small change in the array matrix $\boldsymbol{A}(r, \boldsymbol{\gamma})$ and, in turn, on $\boldsymbol{P}_{\perp}(r, \boldsymbol{\gamma})$. This last feature suggests that the Chebyshev interpolator of the whole matrix $\boldsymbol{P}_{\perp}(r, \gamma)$ may be accurate for a small number of nodes $P$ and over the whole index range (or frequency band) $\left[r_{1}, r_{1}+R-1\right]$.

As we proceed to show, the Chebyshev interpolator of $\boldsymbol{P}_{\perp}(r, \boldsymbol{\gamma})$ is able to exploit these three features in order to reduce the large number of summands $R$ in $L_{M L}(\gamma)$ to a small number $P$. For this, first define the Chebyshev interpolator of $\boldsymbol{P}_{\perp}(r, \gamma)$, by performing the following replacements (or identifications) on (10):

- The equivalent of the variable $\lambda$ is the frequency index $r$, but viewed as a real parameter, (i.e, not necessarily an integer).

- The function's domain is $\left[r_{1}, r_{1}+R-1\right]$, i.e, we identify $a$ with $r_{1}$ and $b$ with $r_{1}+R-1$.

- The equivalent of the linear mapping in $(12)$ is now

$$
\eta_{1}(x) \equiv r_{1}+\frac{R-1}{2}(1-x)
$$

and its inverse is

$$
\eta_{1, i}(r) \equiv 2 \frac{r_{1}-r}{R-1}+1 .
$$

- The function $g(\lambda)$ is now the full matrix $\boldsymbol{P}_{\perp}(r, \boldsymbol{\gamma})$.

- The equivalents of the abscissas $\lambda_{n}$ are denoted $\rho_{n} \equiv$ $\eta_{1}\left(x_{n}\right)$. So the $\rho_{n}$ are the real values of $r$ at which $\boldsymbol{P}_{\perp}(r, \boldsymbol{\gamma})$ is evaluated.

- The weight functions $\phi_{n}(\lambda)$ for $\boldsymbol{P}_{\perp}(r, \gamma)$ are now denoted $\phi_{1, n}(r)$, and can be readily obtained from (18) by replacing $\eta_{i}$ with $\eta_{1, i}$.

These replacements allow us to define the Chebyshev interpolator of $\boldsymbol{P}_{\perp}(r, \gamma)$,

$$
\boldsymbol{P}_{\perp}(r, \boldsymbol{\gamma}) \approx \tilde{\boldsymbol{P}}_{\perp}(r, \boldsymbol{\gamma}) \equiv \sum_{n=1}^{P} \boldsymbol{P}_{\perp}\left(\rho_{n}, \boldsymbol{\gamma}\right) \phi_{1, n}(r),
$$

in which we may expect $P \ll R$. Next, let us substitute this formula into (7):

$$
\begin{gathered}
L_{M L}(\boldsymbol{\gamma})=\sum_{r=r_{1}}^{r_{1}+R-1} \operatorname{tr}\left\{\boldsymbol{P}_{\perp}(r, \boldsymbol{\gamma}) \boldsymbol{R}_{r}\right\} \approx \sum_{r=r_{1}}^{r_{1}+R-1} \operatorname{tr}\left\{\tilde{\boldsymbol{P}}_{\perp}(r, \gamma) \boldsymbol{R}_{r}\right\} \\
=\sum_{r=r_{1}}^{r_{1}+R-1} \operatorname{tr}\left\{\sum_{n=1}^{P} \phi_{1, n}(r) \boldsymbol{P}_{\perp}\left(\rho_{n}, \boldsymbol{\gamma}\right) \boldsymbol{R}_{r}\right\} \\
=\sum_{n=1}^{P} \operatorname{tr}\left\{\boldsymbol{P}_{\perp}\left(\rho_{n}, \boldsymbol{\gamma}\right) \sum_{r=r_{1}}^{r_{1}+R-1} \phi_{1, n}(r) \boldsymbol{R}_{r}\right\} .
\end{gathered}
$$

Finally, defining the correlation matrices

$$
\boldsymbol{R}_{I, n} \equiv \sum_{r=r_{1}}^{r_{1}+R-1} \phi_{1, n}(r) \boldsymbol{R}_{r}
$$


we obtain a new cost function $L_{M L, I}(\gamma)$ with just $P$ summands

$$
L_{M L}(\gamma) \approx L_{M L, I}(\gamma) \equiv \sum_{n=1}^{P} \operatorname{tr}\left\{\boldsymbol{P}_{\perp}\left(\rho_{n}, \boldsymbol{\gamma}\right) \boldsymbol{R}_{I, n}\right\}
$$

We will refer to this formula as the "interpolated DML cost function" in the rest of the paper. The computation method in Sec. III for the Direct DML cost function can be applied to $L_{M L, I}(\gamma)$ without any variation. However, we may expect its computational burden to be much smaller when applied to $L_{M L, I}(\gamma)$ rather than $L_{M L}(\gamma)$, roughly smaller by factor $R / P$. This is so because these two cost functions return the same values, except for the interpolation error $L_{M L}(\gamma)-L_{M L, I}(\gamma)$ that is negligible, already for small values of $P$. As will be shown in Sec. VIII $R / P$ can be a large number.

\section{EXPECTED REDUCTIONS IN COMPLEXITY}

A detailed analysis of the complexity of any wideband DOA estimator is difficult, due to the iterative methods and linesearches involved in them, whose complexity depends on the smoothness of the functions optimized or scanned. We can see this in the estimators in Sec. IIII The $R$ eigendecompositions involved in IC-MUSIC and TOPS implicitly require an iterative method, and the DML estimators are computed by iterating (9) a few times (usually 3 to 5 times). And, as we have already commented, IC-MUSIC, TOPS and DML respectively require line searches on $L_{T}(\gamma), L_{M U}(\gamma)$, and $L_{M L}\left(\left[\gamma_{K, \alpha} ; \gamma\right]\right)$.

We may, however, draw several conclusions from the previous sections:

- The function evaluation method in Sub-sec. IV-C employs a reasonably small number of evaluations of $g(\lambda)$ (those required by the Chebyshev interpolator), and then performs any additional operation through interpolation using the DCT and the barycentric interpolator. Probably, the main complexity reduction lies in the fact that the number of evaluations of $g(\lambda)$ is limited by the number of nodes required by the Chebyshev interpolator, though this obviously depends on the complexity of each evaluation of $g(\lambda)$. We assess the number of such evaluations in the numerical example in Sub-sec. VIII-A.

- The interpolated DML estimator requires an increase of the data processing complexity, given that it is necessary to obtain the compressed matrices $\boldsymbol{R}_{I, n}$ from the matrices $\boldsymbol{R}_{r}$. However, this increase is just a small percentage of the total burden of the data processing. We can check this by evaluating the complexities of the three main data processing operations, which are the FFTs, the computation of the covariance matrices $\boldsymbol{R}_{r}$, and the final computation of the compressed matrices $\boldsymbol{R}_{I, n}$ :

\begin{tabular}{lr|l}
\multicolumn{1}{l|}{ Step } & Cost (complex flops) \\
\hline 1$)$ & DFTs: & $\frac{5}{4} M J N \log _{2} N$ \\
2) & Matrices $\boldsymbol{R}_{r}:$ & $\frac{1}{2} J R M(M+1)+M^{2}(J R-1)$ \\
3) $\boldsymbol{R}_{I, n}$ from $\boldsymbol{R}_{r}:$ & $\frac{1}{2} P\left(R M^{2}+(R-1) M^{2}\right)$ \\
\hline
\end{tabular}

In a typical example, we have $J=100, N=256$, $M=10, R=204$ and $P=6$, and the resulting costs of 1), 2) and 3) in mega flops are, 2.56, 3.16 and 0.122 respectively. Thus, the additional step 3 ) just involves a $2.13 \%$ complexity increase.

- The direct computation of the wideband DML estimator involves a very high complexity, given that $L_{M L}(\gamma)$ has a very large number of summands $R$ (hundreds or thousands). This number must be large because it is proportional to the DFT length $N$ in $(1),(R \approx N B T)$, and $N$ is in turn large in order to obtain a small DFT mismatch. Actually, the implementations in the literature of this estimator attempt to reduce the complexity by considering only the frequencies at which the signal power is high, [13, Sec. 4].

- However, the minimization of the interpolated cost function $L_{M L, I}(\gamma)$ is far less complex, given that it has a small number of summands $P ;(P$ varies from 4 to 7 in the numerical examples for $B \tau_{\max }=0.45$, where $\tau_{\max }$ is the maximum array delay). Actually, using the method in Sub-secs. III-A and III-B, the complexity is reduced by factor $R / P$ if we employ $L_{M L, I}(\gamma)$ rather than $L_{M L}(\gamma)$, because these two functions are the same except for the negligible interpolation error. In the numerical example is Sub-sec. VIII-C it is, at least, $R / P=51$.

- The number of Chebyshev nodes $P$ is roughly proportional to the product $B \tau_{\max }$ and it must be selected to ensure a sufficiently small interpolation error for $\boldsymbol{P}_{\perp}(r, \gamma)$. See Sub-sec. VII.B.

\section{NUMERICAL EXAMPLES}

We have performed several numerical examples following the signal model in Sec. [I] which are presented in the sequel. In them, the main parameters were the following:

Central frequency. The signals' central frequency was $f_{o}=2.4 \mathrm{GHz}$.

Received signals. There were two simulation scenarios, that we term "independent-signals" (IS) and "correlated-signals" (CS). In the IS scenario, the lowpass equivalents of the received signals were three linearly-modulated signals $s_{o, k}(t)$ with raised-cosine modulating pulse (roll-off 0.2 ). The modulation sequences in the three were variance-one independent and complex white. The corresponding lowpass signals at the sensor array reference point were $a_{k}^{\prime} s_{o, k}\left(t-\tau_{k}^{\prime}\right)$, with

$$
\begin{gathered}
\text { Amplitudes } a_{k}^{\prime}: 0.626+j 0.7798,-0.4432-j 0.552, \\
0.3138+j 0.3908,
\end{gathered}
$$

Delays $\tau_{k}^{\prime} /\left(2 f_{o}\right): 0,0.6,37.53$.

In the CS scenario, the lowpass equivalents were generated as in the IS scenario, except for the fact that the three initial signals $s_{o, k}(t)$ were the same one.

Signals' bandwidths relative to the maximum array delay. All signals had two-sided bandwidth $B$. The product of $B$ with the maximum array delay $\tau_{\max }$ was $B \tau_{\max }=0.45$.

DFT length and number of slots. The DFT length was $N=256$ and the number of slots was $J=100$. The sampling period $T$ followed $B T=0.8$. The frequency index range was $\left[r_{1}, r_{1}+R-1\right]=[-102,102]$, where index 0 was exactly placed at frequency $f_{o}$. 
Sensor array. Nonuniform linear array with $M=10$ sensors and isotropic patterns, $\left[b_{m}(\gamma)=1\right.$ for all sensors]. The sensors were placed in two co-linear arrays formed by 5 sensors each. Their positions were $m \lambda$ and $(m+0.4) \lambda$, $m=0,1, \ldots, 4$, where $\lambda$ denotes the wavelength.

Angles of arrival. $-0.7895,-0.6816$, and 0.2734 rads. Corresponding parameters in $\gamma:-0.71,-0.63$, and 0.27 .

Signal-to-noise ratio (SNR). Ratio of the signal and noise powers in (1), averaged over all the values of $r$ and $m$ in that equation.

Estimators. We have evaluated four estimators:

- IC-MUSIC.

- TOPS.

- Direct DML. DML estimator computed by minimizing $L_{M L}(\gamma)$ using the method in Sub-secs. III-A and III-B

- $D M L-P$. Interpolated DML estimator computed by minimizing $L_{M L, I}(\gamma)$ for a specific number of interpolation nodes $P$ and using the method in Sub-secs. III-A and III-B

Number of Monte Carlo trials. The estimators' performances were evaluated in 1000 Monte Carlo trials.

\section{A. Interpolation of one-dimensional pseudo-spectra}

The performances of the Chebyshev interpolator for the functions $L_{M U}(\gamma), L_{T}(\gamma)$, and $L_{M L}\left(\left[\gamma_{K, \alpha} ; \gamma\right]\right)$ are similar and, therefore, we present in this section a numerical evaluation of $L_{M U}(\gamma)$ only.

Fig. 1(a) shows a realization of the IC-MUSIC pseudospectrum and its Chebyshev approximation for number of nodes $P=20$ and 40 . In this figure, the mismatch between the exact pseudo-spectrum and its approximation for $P=20$ is clear, though the interpolator is able to follow the main variations of the pseudo-spectrum. For $P=40$ (crosses ' + '), the interpolation error is too small to be visible. Fig. 1 (b) shows this last error (difference between the exact and the interpolated pseudo-spectra for $P=40$ ). Comparing the vertical axis of Figs. 1(a) and 1(b), we can readily see that the $P=40$ interpolator roughly gives between 5 and 6 decimal digits of accuracy.

Fig. 1(c) shows the relative interpolation error versus the number of Chebyshev nodes $P$. In this figure, the relative error is defined as the quotient between the maximum absolute error for $\gamma$ in $[-1,1]$ and the pseudo-spectrum's maximum amplitude. Note the exponential error decrease for a sufficiently large $P$. In the simulations performed for the next section, $P$ was set equal to 50 for the three functions $L_{M U}(\gamma), L_{T}(\gamma)$, and $L_{M L}\left(\left[\gamma_{K, \alpha} ; \gamma\right]\right)$, which is a conservative choice.

\section{B. Projection matrix interpolation performance}

Fig. 2 shows the Chebyshev interpolation error in the approximation of the whole projection matrix $\boldsymbol{P}_{\perp}(r, \gamma)$ for several values of $P$. The error measure in this figure is

$$
\frac{\max _{r, m, m^{\prime}}\left|\left[\boldsymbol{P}_{\perp}(r, \gamma)-\tilde{\boldsymbol{P}}_{\perp}(r, \boldsymbol{\gamma})\right]_{m, m^{\prime}}\right|}{\max _{r, m, m^{\prime}}\left|\left[\boldsymbol{P}_{\perp}(r, \boldsymbol{\gamma})\right]_{m, m^{\prime}}\right|},
$$

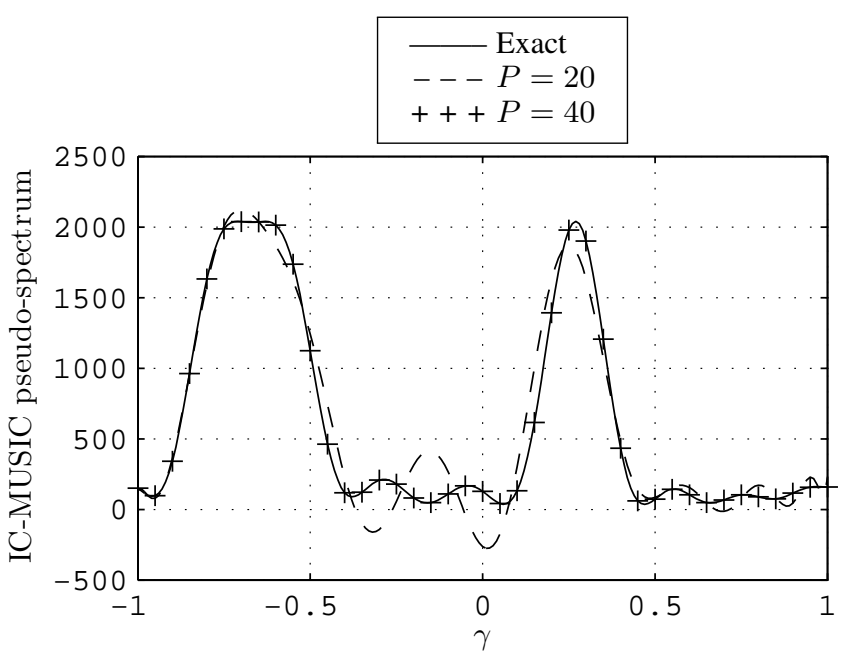

(a) IC-MUSIC pseudo-spectrum and its Chebyshev approximations for $P=20$ and 40 .

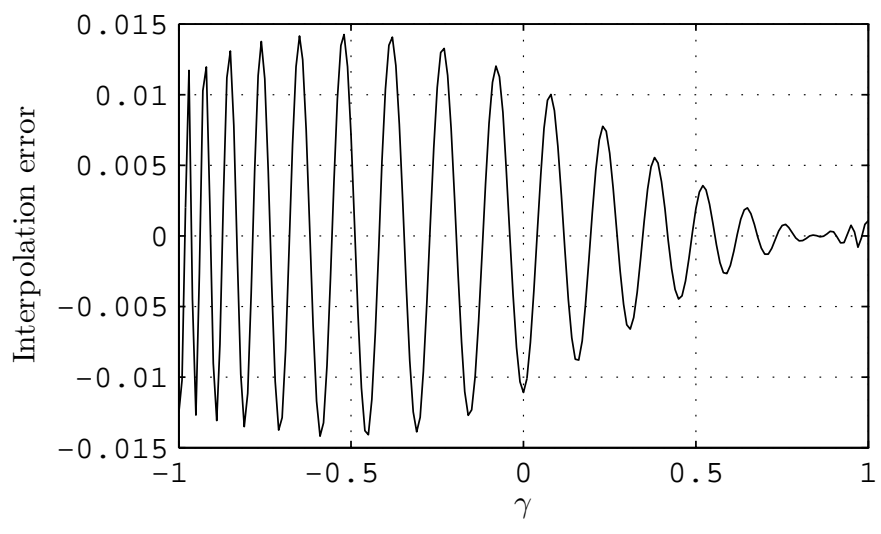

(b) Pseudo-spectrum interpolation error for $P=40$.

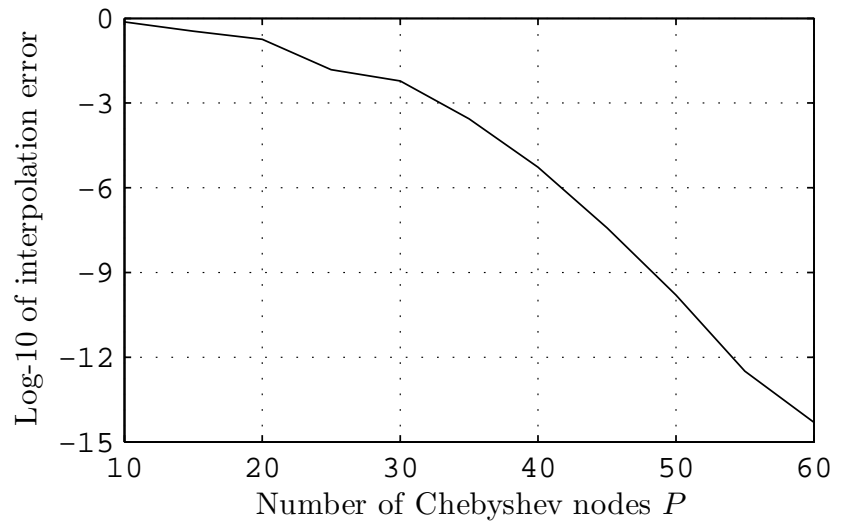

(c) Log-10 of interpolation error versus the number of interpolation nodes $P$.

Fig. 1. Chebyshev interpolation of the IC-MUSIC pseudo-spectrum. (a) Exact pseudo-spectrum and interpolated ones for $P=20$ and 40. (b) Absolute interpolation error for $P=40$. (c) Relative interpolation error versus number of Chebyshev nodes $P$. 


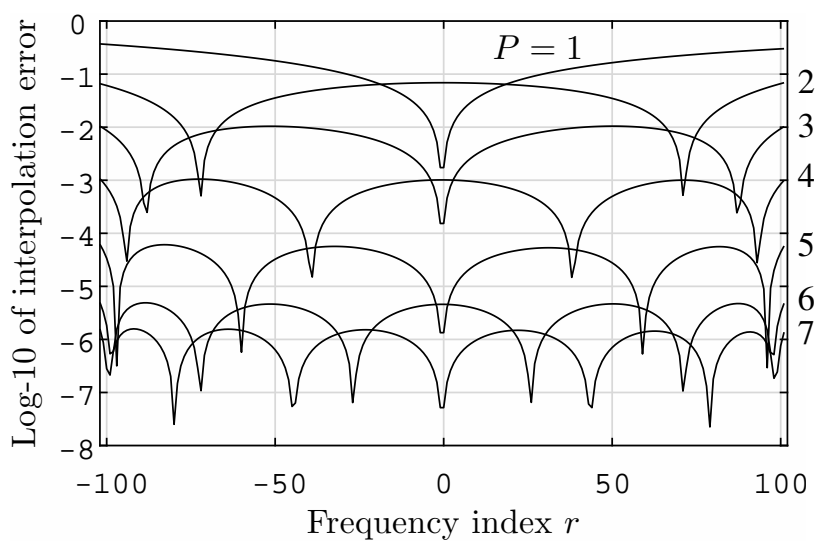

Fig. 2. Chebyshev interpolation error for the projection matrix versus the frequency index $r$ for several interpolation orders $P$.

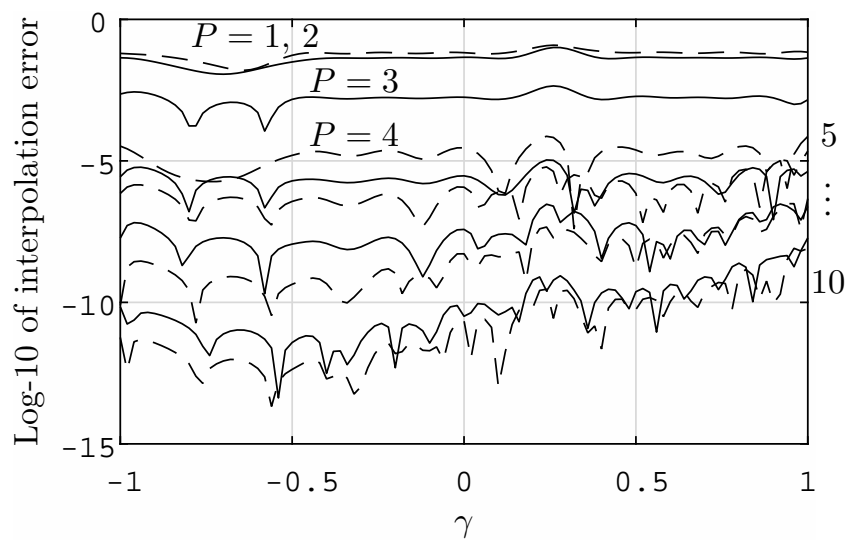

Fig. 3. Error in approximating $L_{M L}([-0.71 ; \gamma])$ with $L_{M L, I}([-0.71 ; \gamma])$. Increasing $P$ shifts the curve downward and the curves for odd $P$ are discontinuous.

where the maxima are taken for indices $r, m$, and $m^{\prime}$ varying in ranges $r_{1} \leq r \leq r_{1}+R-1$ and $1 \leq m, m^{\prime} \leq M$ respectively. As can be readily seen in this figure, the Chebyshev interpolation error decreases very fast with $P$. Actually, $P=6$ already gives 5 digits of accuracy.

Fig 3 presents the error in approximating $L_{M L}(\gamma)$ with $L_{M L, I}(\gamma)$ in one specific example. Specifically, this figure shows the error measure

$$
\frac{\left|L_{M L}([-0.71 ; \gamma])-L_{M L, I}([-0.71 ; \gamma])\right|}{\left|L_{M L}([-0.71 ; \gamma])\right|}
$$

in approximating $L_{M L}([-0.71 ; \gamma])$ with $L_{M L, I}([-0.71 ; \gamma])$ for varying $\gamma$ and several values of $P$. Note that the error decreases fast with $P$.

\section{Effect of the projection matrix interpolation on the estima- tion performance}

In this sub-section, we evaluate the effect of the projection matrix interpolation on the estimation performance. We start assuming incoherent signals (IC scenario). Fig. 4 shows the RMS error in the estimation of the first component of $\gamma$ using IC-MUSIC and TOPS, and Fig. 5(a) shows the same error for Direct DML and DML-P. These last estimations were

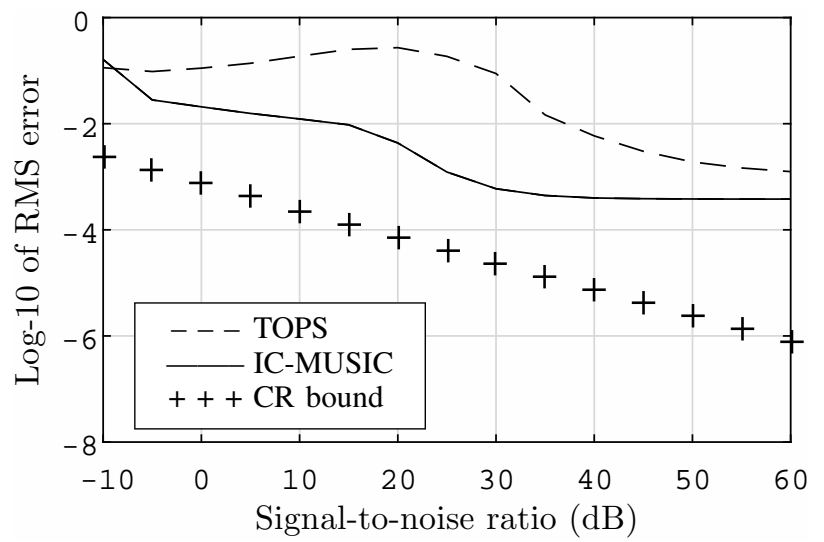

Fig. 4. Log-10 of RMS error in the estimation of $\gamma_{1}$ for IC-MUSIC and TOPS, an Cramer Rao (CR) bound.

\begin{tabular}{l|l} 
Estimator & Timing $(\mathrm{sec})$. \\
\hline IC-MUSIC & 0.228 \\
TOPS & 0.303 \\
Direct DML & 2.823 \\
DML-1 & 0.09703 \\
DML-2 & 0.1094 \\
DML-4 & 0.1254 \\
DML-5 & 0.1416 \\
DML-6 & 0.1558 \\
DML-7 & 0.1714
\end{tabular}

TABLE I

COMPUTATION TIMES OF THE ESTIMATORS IN SECONDS FOR THE EXPERIMENT CORRESPONDING TO FIG.4, AVERAGED OVER 100 TRIALS.

computed using the method in Sub-secs. III-A and III-B. In Fig. 5(a), the curves have been labeled with the corresponding value of $P$. In Fig. 4, it is clear that IC-MUSIC and TOPS are not efficient statistically [do not reach the Cramer-Rao (CR) bound for high SNRs] and, comparing Fig. 4 with 5(a), it is also clear the the DML estimators perform far better than ICMUSIC and TOPS. Figs. 5(b) and 5(c) show the RMS error of the DML estimators for the other two components of $\gamma$, $\gamma_{2}$ and $\gamma_{3}$. The conclusions are similar to those for the first component. Note that in Figs. 5(a) to 5(c) the RMS error for $P=1$ may be either smaller or larger than for $P=2$, for different components of $\gamma$. This phenomenon is a consequence of the poor and similar accuracy achievable for $P=1$ and $P=2$ [Fig. 3], that produces a high bias, which in turn is the main component of the RMS error at high SNRs.

The performance of DML- $P$ is roughly the same as that of the direct DML method already for $P=4$. Note that this implies a very large complexity reduction, given that the DML cost function has $R=204$ summands while DML-4 only has $P=4$, i.e, the complexity reduction is roughly factor $R / P=$ $204 / 4=51$.

The DML-1, DML-2 and DML-3 have a high RMS floor in Figs 5(a) to 5(c), This behavior is caused by the interpolation error given that, for too low values of $P$, the cost function is not interpolated with enough accuracy (Fig. 3), and this translates into a bias.

Another phenomenon is the floor reached by the DML estimators at high SNRs, that seems to contradict the statistical efficiency of the DML estimator. However, this floor is just 


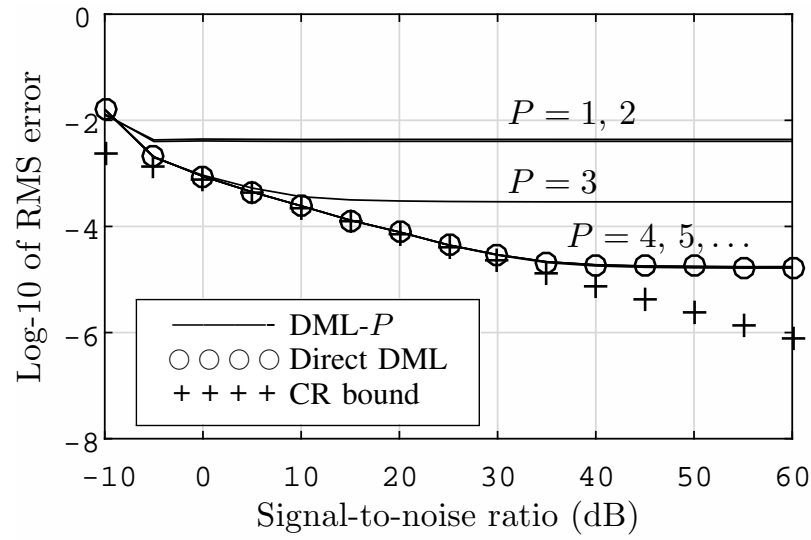

(a) Log-10 of RMS error in the estimation of $\gamma_{1}$ for Direct DML and DML- $P$, and Cramer Rao $(\mathrm{CR})$ bound. $(N=256, J=100$.)

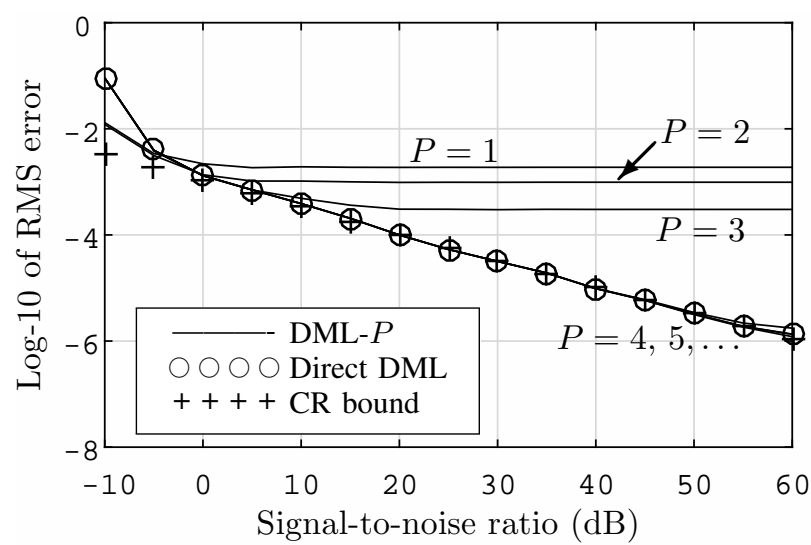

(b) Log-10 of RMS error in the estimation of $\gamma_{2}$ for Direct DML and DML- $P$, and Cramer Rao $(\mathrm{CR})$ bound. $(N=256, J=100$.)

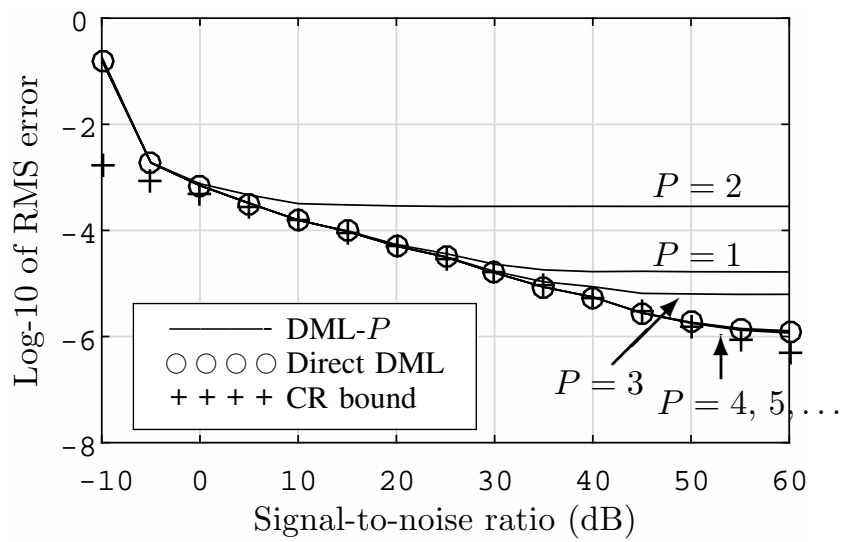

(c) Log-10 of RMS error in the estimation of $\gamma_{3}$ for Direct DML and DML- $P$, and Cramer Rao (CR) bound. ( $N=256, J=100$.)

Fig. 5. RMS error performance of Direct DML and DML- $P$ in the estimation of $\gamma_{1}, \gamma_{2}$, and $\gamma_{3}$. (a), (b), and (c) correspond to $\gamma_{1}, \gamma_{2}$, and $\gamma_{3}$ respectively.

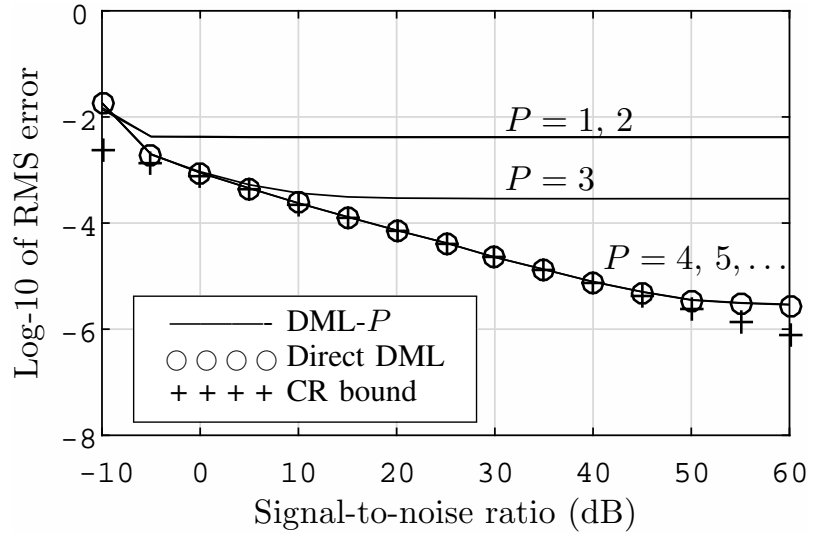

(a) Repetition of Fig. 5(a) but for DFT length $N=1024$ and $J=25$ slots.

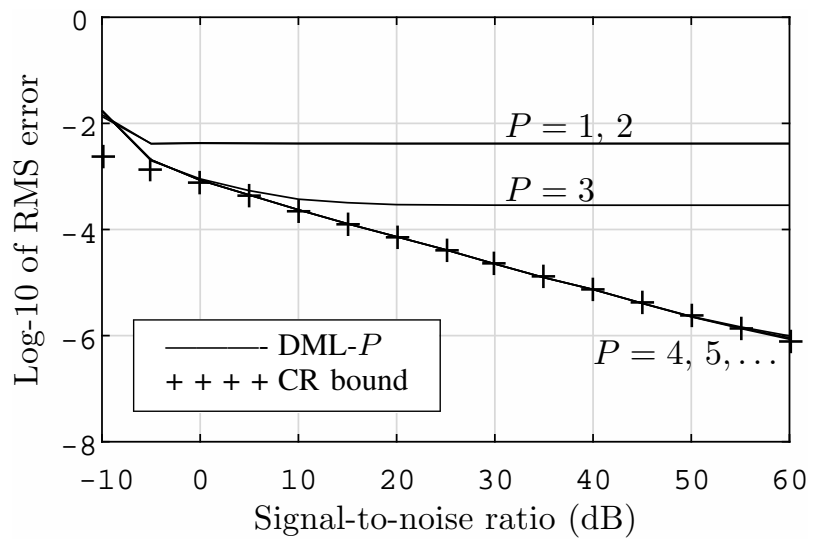

(b) Repetition of Fig. 5(a) but for DFT length $N=25600$ and $J=1$ slot.

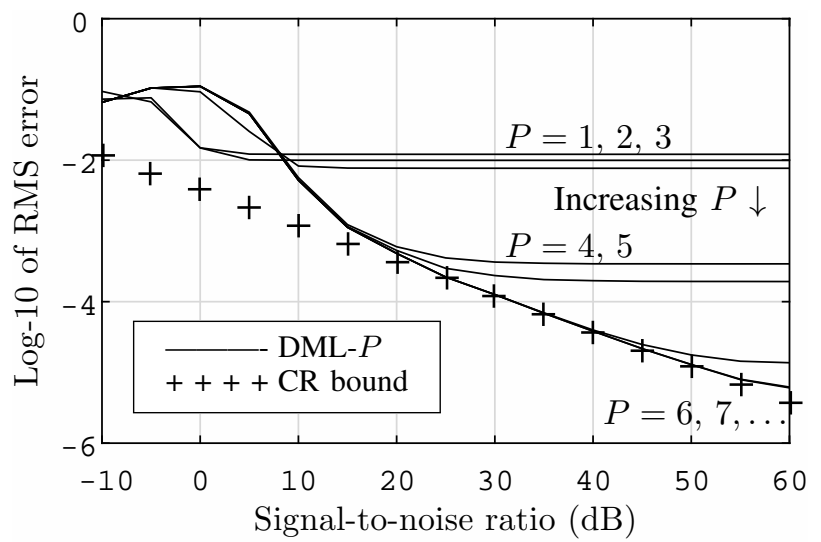

(c) Repetition of Fig. 5(a) but in the CS scenario and for DFT length $N=25600$ and $J=1$ slot.

Fig. 6. Repetition of Fig. 5(a) for (a) IS scenario, $N=1024$ and $J=25$; (b) IS scenario, $N=25600$ and $J=1$; and (c) CS scenario, $N=25600$ and $J=1$. 
the mismatch produced by the too-small FFT length, since it is only $N=256$. Fig. 6(a) is a repetition of Fig. 5(a) but for DFT length $N=1024$ and $J=25$ slots. Note that the floor appears at higher SNRs than in Fig. 5(a). Fig. 6(b) is another repetition of Fig. 5(a) but with the highest DFT length, $N=25600, J=1$. Here the mismatch is negligible. Note that, in this last case, IC-MUSIC and TOPS are not usable, given that there is a single snapshot and, besides, the Direct DML estimator is intractable, because its cost function has $R=20480$ summands, while DML-4 gives the same performance with just $P=4$ summands. So, the compression ratio of the DML cost function is $R / P=5120$.

Table I shows the computation times for a single estimate, averaged over 100 trials. Note that Direct DML is the one taking longer, due to the many terms in its cost function. The timings for the DML- $P$ estimates follow a linear trend approximatey, whose least squares fit is

$$
\text { Timing } \approx 0.08143+0.01482 P(\mathrm{sec}) .
$$

This linear fit suggests that the time required for computing the DML estimate is $0.08143 \mathrm{sec}$, while the rest of the time is consumed in evaluating the cost function. Also, it suggests that each additional node involves an increase of $0.01482 \mathrm{sec}$. The computation times of IC-MUSIC and TOPS are larger than those of the DML- $P$ estimates.

Finally, we evaluate the CS scenario. As is well-known, ICMUSIC and TOPS are not applicable to coherent signals, and the conclusions for the Direct DML and DML- $P$ estimators are the same as in the IS scenario. Fig. 6(c) is a repetition of Fig. 6(b) but in the CS scenario, $(N=25600, J=1)$.

\section{CONCLUSIONS}

We have presented a function evaluation method applicable to the usual line-search tasks in DOA estimation, both narrowband and wideband. The method is a combination of the Chebyshev and barycentric interpolators, and employs the DCT. It allows one to reduce the number of function evaluations required in the usual line-search tasks. A second contribution is a method to compress the DML cost function, so that it is formed by just a few narrowband-like components, with the associated reduction in complexity. The methods in the paper have been assessed numerically.

\section{REFERENCES}

[1] Harry L. van Trees, Detection, Estimation, and Modulation Theory. Part IV, Optimum array processing, John Wiley \& Sons, Inc, first edition, 2002.

[2] H. Wang and M. Kaveh, "Coherent signal-subspace processing for the detection and estimation of angles of arrival of multiple wideband sources," IEEE Transactions on Acoustics, Speech, and Signal Processing, vol. 33, no. 4, pp. 823-831, Aug 1985.

[3] S. Valaee and P. Kabal, "Wideband array processing using a two-sided correlation transformation," IEEE Transactions on Signal Processing, vol. 43, no. 1, pp. 160-172, Jan 1995.

[4] T. K. Yasar and T. E. Tuncer, "Wideband DOA estimation for nonuniform linear arrays with Wiener array interpolation," in 2008 th IEEE Sensor Array and Multichannel Signal Processing Workshop, July 2008, pp. 207-211.

[5] W. J. Zeng and X. L. Li, "High-resolution multiple wideband and nonstationary source localization with unknown number of sources," IEEE Transactions on Signal Processing, vol. 58, no. 6, pp. 3125-3136, June 2010.
[6] M. Wax, Tie-Jun Shan, and T. Kailath, "Spatio-temporal spectral analysis by eigenstructure methods," IEEE Transactions on Acoustics, Speech, and Signal Processing, vol. 32, no. 4, pp. 817-827, Aug 1984.

[7] Yeo-Sun Yoon, L. M. Kaplan, and J. H. McClellan, "TOPS: new DOA estimator for wideband signals," IEEE Transactions on Signal Processing, vol. 54, no. 6, pp. 1977-1989, June 2006.

[8] A. K. Shaw, "Improved wideband DOA estimation using modified TOPS (mTOPS) algorithm," IEEE Signal Processing Letters, vol. 23, no. 12, pp. 1697-1701, Dec 2016.

[9] Sathish Chandran, Ed., Advances in Direction-of-Arrival Estimation, Artech House, 2006.

[10] Engin Tuncer and Benjamin Friedlander, Eds., Classical and modern direction-of-arrival estimation, Elsevier, 2009.

[11] M. A. Doron, A. J. Weiss, and H. Messer, "Maximum-likelihood direction finding of wide-band sources," IEEE Transactions on Signal Processing, vol. 41, no. 1, pp. 411-414, Jan 1993.

[12] Lean Yip, Joe C. Chen, Ralph E. Hudson, and Kung Yao, "Cramer-Rao bound analysis of wideband source localization and DOA estimation," in International Symposium on Optical Science and Technology. International Society for Optics and Photonics, 2002, pp. 304-316.

[13] L. Yip, C. E. Chen, R. E. Hudson, and K. Yao, "DOA estimation method for wideband color signals based on least-squares joint approximate diagonalization," Proceedings of Sensor Array and Multichannel Signal Processing, pp. 104-107, 2008.

[14] Joe C. Chen, Ralph E. Hudson, and Kung Yao, "Maximum-likelihood source localization and unknown sensor location estimation for wideband signals in the near-field," IEEE Transactions on Signal Processing, vol. 50, no. 8, pp. 1843-1854, 2002.

[15] Q. Shen, W. Liu, W. Cui, S. Wu, Y. D. Zhang, and M. G. Amin, "Group sparsity based wideband DOA estimation for co-prime arrays," in 2014 IEEE China Summit International Conference on Signal and Information Processing (ChinaSIP), July 2014, pp. 252-256.

[16] Q. Shen, W. Liu, W. Cui, S. Wu, Y. D. Zhang, and M. G. Amin, "Low-complexity direction-of-arrival estimation based on wideband coprime arrays," IEEE/ACM Transactions on Audio, Speech, and Language Processing, vol. 23, no. 9, pp. 1445-1456, Sept 2015.

[17] Petros T. Boufounos, Paris Smaragdis, and Bhiksha Raj, "Joint sparsity models for wideband array processing," Proceedings SPIE, vol. 8138, pp. 1-10, 2011.

[18] Ralph O. Schmidt, "Multiple emitter location and signal parameter estimation," IEEE Transactions on and Antennas Propagation, vol. 34, no. 3, pp. 276-280, Mar. 1986.

[19] B. Ottersten, M. Viberg, P. Stoica, and A. Nehorai, "Exact and large sample maximum likelihood techniques for parameter estimation and detection in array processing," in Radar Array Processing, S. Haykin, J. Litva, and A. Nehorai, Eds., chapter 4, pp. 99-151. Springer-Verlag, Berlin, 1993.

[20] John C. Mason and David C. Handscomb, Chebyshev polynomials, CRC Press, 2002.

[21] J. Selva, "Design of barycentric interpolators for uniform and nonuniform sampling grids," IEEE Transactions on Signal Processing, vol. 58, no. 3, pp. $1618-1627$, Mar 2010.

[22] I. Ziskind and M. Wax, "Maximum likelihood localization of multiple sources by alternating projection," IEEE Transactions on Acoustics, Speech, and Signal Processing, vol. 36, pp. 1553-1560, Oct. 1988.

[23] J. Selva, "ML estimation and detection of multiple frequencies through periodogram estimate refinement," IEEE Signal Processing Letters, vol. 24, no. 3, pp. 249-253, March 2017.

[24] T. V. Ho, J. G. McWhirter, A. Nehorai, U. Nickel, B. Ottersten, B. D. Steinberg, P. Stoica, M. Viberg, Z. Zhu, S. Haykin, et al., Radar array processing, vol. 25, Springer Science \& Business Media, 2013.

[25] Mats Viberg, Björn Ottersten, and T. Kailath, "Detection and estimation in sensor arrays using weighted subspace fitting," IEEE Transactions on Signal Processing, vol. 39, no. 11, pp. 2436-2449, Nov. 1991.

[26] L. Kaufman, "A variable projection method for solving separable nonlinear least squares problems," BIT, vol. 15, pp. 49-57, 1975.

[27] J. Selva, "An efficient Newton-type method for the computation of ML estimators in a Uniform Linear Array," IEEE Transactions on Signal Processing, vol. 53, no. 6, pp. 2036-2045, June 2005.

[28] J. E. Dennis Jr. and Robert B. Schnabel, Numerical Methods for Unconstrained Optimization and Nonlinear Equations, Number 16 in Classics in Applied Mathematics. SIAM, 1996.

[29] Donald Fraser, "Interpolation by the FFT revisited-an experimental investigation," IEEE Transactions on Acoustics, Speech, and Signal Processing, vol. 37, no. 5, pp. 665-675, May 1989. 
[30] J. J. Knab, "Interpolation of band-limited functions using the Approximate Prolate series," IEEE Transactions on Information Theory, vol. IT-25, no. 6, pp. 717-720, Nov 1979. 